\title{
Epidermal growth factor receptor protein expression and gene amplification in the chemorefractory metastatic embryonal carcinoma
}

\author{
Xiaoyan Wang ${ }^{1}$, Shaobo Zhang ${ }^{1}$, Gregory T MacLennan ${ }^{2}$, Katharina Biermann ${ }^{1,3}$, \\ Richard S Foster ${ }^{4}$, Stephen D Beck ${ }^{4}$ and Liang Cheng ${ }^{1,4}$ \\ ${ }^{1}$ Department of Pathology and Laboratory Medicine, Indiana University School of Medicine, Indianapolis, IN, \\ USA; ${ }^{2}$ Department of Pathology, Case Western Reserve University, Cleveland, OH, USA; ${ }^{3}$ Erasmus MC, \\ University Medical Center Rotterdam, Josephine Nefkens Institute, Rotterdam, The Netherlands and \\ ${ }^{4}$ Department of Urology, Indiana University School of Medicine, Indianapolis, IN, USA
}

\begin{abstract}
Testicular cancer is the most common cancer in young male patients. The combination of cisplatin-based chemotherapy and surgery has resulted in high cure rates. However, a small percentage of patients have cancers that are refractory to chemotherapy; treatment options for these patients are limited, and their prognosis is generally poor. Further studies are needed to explore the molecular pathogenesis pathways and potential targets of therapy for this group of highly aggressive tumors. We analyzed 21 patients who presented with metastatic embryonal carcinoma, were treated with chemotherapy, and subsequently underwent retroperitoneal lymph node dissection. Immunostaining for epidermal growth factor receptor (EGFR) was performed on paraffin-embedded tissue sections containing tumor from these specimens, using the avidinbiotin-peroxidase method. EGFR gene amplification was performed by interphase fluorescence in situ hybridization (FISH). Immunohistochemically, 9 of 21 cases (43\%) demonstrated positive EGFR staining; 12 of 21 cases $(57 \%)$ had absent or very limited EGFR expression. FISH revealed EGFR amplification in 1 case $(5 \%)$, polysomy in 15 of 21 cases $(71 \%)$, and normal disomy pattern in 5 of 21 cases $(24 \%)$. A significant correlation between EGFR protein expression level and its chromosome polysomy/amplification was established $(P=0.02)$. Our study showed that EGFR protein is frequently expressed in a subset of patients with chemorefractory metastatic embryonal carcinoma. EGFR chromosomal polysomy/amplification may be one of the mechanisms that cause increased EGFR protein expression, and could potentially be used as indication for anti-EGFR therapy.
\end{abstract}

Modern Pathology (2009) 22, 7-12; doi:10.1038/modpathol.2008.133; published online 25 July 2008

Keywords: testis; germ cell tumor; metastatic embryonal carcinoma; epidermal growth factor receptor; molecular markers; prognosis

Testicular cancer is the most common cancer in male patients between 15 and 35 years of age. ${ }^{1}$ Germ cell tumors are divided into seminoma and nonseminomatous germ cell tumors based on histological, biological, and clinical features. Several histological factors have been shown to predict the risk of metastasis or relapse of disease, including

Correspondence: Dr L Cheng, MD, Department of Pathology and Laboratory Medicine, Indiana University School of Medicine, 350 West 11th Street, Clarian Pathology Laboratory Room 4010, Indianapolis, IN 46202, USA.

E-mail: liang_cheng@yahoo.com

Received 31 March 2008; revised 30 June 2008; accepted 02 July 2008; published online 25 July 2008 vascular invasion, presence or volume of embryonal carcinoma component, and absence of yolk sac tumor. The combination of surgery- and cisplatinbased chemotherapy has resulted in cure rates of 70 to $>95 \%$ in nonseminomatous germ cell tumors, depending on the stage of the tumor. ${ }^{2,3}$ However, a small group of patients who have late relapse or are refractory to chemotherapy have few treatment options and are faced with a poor prognosis.

Epidermal growth factor receptor $(E F G R)$ gene, one of the oncogenes on chromosome 7 , has been implicated in the pathogenesis and progression of many malignancies. ${ }^{4}$ However, its role in the pathogenesis of germ cell tumors has not been extensively investigated. Overexpression of EGFR 
in various types of carcinoma has prompted the development of agents directed against EGFR. ${ }^{5}$ Targeted therapies against EGFR have been successfully used in the treatment of some advanced colorectal and non-small-cell lung cancers. ${ }^{6-8}$ In this study, we examined EGFR protein expression by immunohistochemical staining and assessed gene amplification by fluorescence in situ hybridization (FISH) in tumorous tissue from 21 patients with metastatic chemorefractory embryonal carcinoma, and also investigated correlations between EGFR protein expression and gene amplification in this tumor. Our study was designed to provide further insights into the molecular pathogenesis of metastatic germ cell tumor and to help define potential therapies for this uncommon malignancy.

\section{Materials and methods}

\section{Patients}

A retrospective review of Indiana University Medical Center testis cancer database of 1990-2002 was performed to identify patients who presented with metastatic nonseminomatous germ cell tumor, were treated with three or four courses of cisplatin-based chemotherapy, and subsequently underwent retroperitoneal lymph node dissection (RPLND) at the Indiana University Medical Center. A total of 21 patients (age range: 19-40 years, mean age: 32 years) with residual metastatic embryonal carcinoma in their RPLND specimens were identified, and tumor tissue from their specimens was evaluated in this study. Formalin-fixed, paraffin-embedded tissue from RPLND was available for all cases.

Formalin-fixed, paraffin-embedded tissue was available in only two cases of primary testicular tumors, including one teratoma and one embryonal carcinoma. We were not able to retrieve other primary testicular tumors for analysis as most orchiectomies were performed at the outside institutions during the period of 1979-1996.

\section{Immunohistochemical Analysis}

Immunohistochemical staining for EGFR was performed on formalin-fixed and paraffin-embedded tissue sections using the peroxidase-labeled streptavidin-biotin method. Tissue sections $(5 \mu \mathrm{m})$ from each patient were used for EGFR immunohistochemical staining. Sections were deparaffinized in xylene for $5 \mathrm{~min}$ and then rehydrated through graded ethanol to distilled water. Antigen retrievel was performed by heating sections for $15 \mathrm{~min}$ (Target Retrievel; Dako, Carpinteria, CA, USA). Endogenous peroxidase was blocked by incubation in $3 \%$ $\mathrm{H}_{2} \mathrm{O}_{2}$ for $5 \mathrm{~min}$. Tissue sections were incubated with primary antibodies against EGFR (Dako) for
$10 \mathrm{~min}$, followed by biotinylated secondary antibody (Dako) and peroxidase-labeled streptavidin (Dako). 3,3-Diaminobenzidine was used as the chromogen. The extent of staining was evaluated by visual examination microscopically. Each section was scanned at low magnification for membranous staining and was scored by evaluating the percentage of tumor cells staining positively. According to the scoring system recommended by Tsao et al, ${ }^{9}$ results were recorded as positive expression $(\geq 10 \%$ of cells staining) or negative expression $(<10 \%$ of cells staining).

\section{Tissue Preparations and FISH}

Sections (4- $\mu$ m-thick) were cut from paraffin blocks from each patient. The slides were deparaffinized with two washes of xylene, $15 \mathrm{~min}$ each, and subsequently washed twice with absolute ethanol, $10 \mathrm{~min}$ each and then air-dried in the hood. Next, the slides were treated in $0.1 \mathrm{mM}$ citric acid ( $\mathrm{pH}$ 6.0) (Zymed, CA, USA) at $95^{\circ} \mathrm{C}$ for $10 \mathrm{~min}$, rinsed in distilled water for $3 \mathrm{~min}$ followed by a wash of $2 \times$ SSC (standard saline citrate) for 5 min. Digestion of the tissue was performed by applying $0.4 \mathrm{ml}$ of pepsin $(5 \mathrm{mg} / \mathrm{ml}$ in $0.1 \mathrm{~N} \mathrm{HCl}$ with $0.9 \% \mathrm{NaCl})$ (Sigma, St Louis, MO, USA) at $37^{\circ} \mathrm{C}$ for $40 \mathrm{~min}$. The slides were rinsed with distilled water for $3 \mathrm{~min}$, and then washed with $2 \times$ SSC for $5 \mathrm{~min}$ and air-dried.

EGFR gene chromosomal alterations was detected by interphase FISH using LSI EGFR/CEP 7 Dual Color Probe containing centromere chromosome 7 (Centromeric Enumeration Probe, CEP 7, Spectrum Green) and EGFR located at 7p12 (EGFR, Spectrum Orange) (Vysis, Downers Grove, IL, USA). The probe was diluted with $t$ DenHyb2 (Insitus, Albuquerque, NM, USA) in a ratio of 1:50. Diluted probe ( $5 \mu \mathrm{l})$ were applied to each slide in reduced light conditions; the slides were then covered with a $22 \times 22 \mathrm{~mm}$ coverslip and sealed with rubber cement. Denaturation was achieved by incubating the slides at $80^{\circ} \mathrm{C}$ for $10 \mathrm{~min}$ in a humidified box, followed by hybridization at $37^{\circ} \mathrm{C}$ over night. The coverslip was removed and the slides were washed extensively with two $45^{\circ} \mathrm{C}$ prewarmed $0.1 \times \mathrm{SSC} /$ $1.5 \mathrm{M}$ urea, $20 \mathrm{~min}$ for each, followed by a wash with $2 \times$ SSC for $20 \mathrm{~min}$ and a $2 \times$ SSC/0.1\% NP40 for $10 \mathrm{~min}$ at $45^{\circ} \mathrm{C}$. The slides were further washed with room temperature $2 \times$ SSC for $5 \mathrm{~min}$ and air-dried. The slides were counterstained with $10 \mu \mathrm{l}$ 4-6diamidino-2-phenylindole (DAPI; Insitus), covered with coverslips and sealed with nail polish.

The slides were examined using a Zeiss Axioplan 2 microscope (Zeiss, Göttingen, Germany) with the following filters: SP-100 DAPI, FITC MF-101 for Spectrum Green (CEP 7) and Gold 31003 for Spectrum orange (EGFR) from Chroma (Chroma, Brattleboro, VT, USA). The images were acquired with a CCD camera and analyzed 
with MetaSystem Isis Software (MetaSystem, Belmont, MA, USA). Four sequential focus stacks with $0.4-\mu \mathrm{m}$ intervals were acquired and then integrated into a single image to reduce thickness-related artifacts.

\section{FISH Analysis}

The method of analysis was as previously described in detail. ${ }^{10,11}$ In brief, for each slide, 100 neoplastic nuclei were scored for signals from both CEP7 and EGFR probes under the fluorescence microscope with $\times 1000$ magnification. Signals from nonoverlapping nuclei were enumerated. Two signals of the same size in close proximity, not connected by a link, were counted as two signals. A diffuse signal was regarded as a signal if it was contiguous and within an acceptable boundary. Two small signals connected by a visible link were counted as one signal. Overlapping nuclei and nuclei with uncertain signal were not included. In accordance with the criteria of Alvarez et $a{ }^{12}{ }^{12}$ EGFR amplification was considered to be present if $>10 \%$ of the nuclei contained multiple EGFR signals and the EGFR/ CEP7 ratio was $>2 .{ }^{10,11}$ Polysomy for an individual tumor was defined as follows: $>10 \%$ of the nuclei contained $>2$ signals of CEP7 or EGFR and the EGFR/CEP7 ratio was $<2$. Individual tumors were considered to show disomy if the percentage of disomic cells (with two copies of EGFR and CEP7) fell into the range of summarized average \pm three standard deviations of the normal control cells and did not meet the criteria for amplification or polysomy. For each FISH preparation, known positive and negative cells were used as controls. Preparations were considered valid if $>90 \%$ of the cells showed bright signals. ${ }^{10,11}$

\section{Statistical Analysis}

Data were analyzed using SAS version 9.1 (SAS Institute, Cary, NC, USA). Correlations between EGFR protein expression and gene amplification were evaluated by using Cochran-Mantel-Hanszel. A $P$-value $<0.05$ was considered significant, and all $P$-value tests were two sided.

\section{Results}

\section{Clinical and Pathological Features}

In all, 21 patients (age range: $19-40$ years, mean age: 32 years) were found to have residual metastatic embryonal carcinoma in their RPLND specimens, despite prior treatment with cisplatin-based chemotherapy. In 6 cases, the residual tumor was pure embryonal carcinoma; in the other 15 cases, the residual tumors contained two or more different histological elements. The percentage of each component in these 21 tumors was as follows: embryonal carcinoma (present in 21 of 21 tumors, $100 \%$ ), teratoma (7 of $21,33 \%$ ), yolk sac tumor $(7$ of $21,33 \%)$ and choriocarcinoma (3 of $21,14 \%$ ).

\section{Immunohistochemical Study of EGFR Protein Expression}

Immunohistochemical evidence of EGFR protein expression was evaluated in the metastatic embryonal components of all 21 cases. Positive immunohistochemical expression of EGFR was observed in tissue sections from 9 of 21 patients (43\%), 1 with $10-25 \%$ staining, 4 with $25-50 \%$ staining, 1 with $50-75 \%$ staining, and 3 with $75-100 \%$ staining (Figure 1). By contrast, immunohistochemical expression of EGFR was absent or very limited in 12 of 21 cases (57\%), 11 with no staining, and 1 with $1-10 \%$ staining.

We also performed EGFR immunostaining on two primary testicular tumors available (one teratoma and one embryonal carcinoma). Both primary tumors showed negative immunostainings for EGFR. Corresponding retroperitoneal metastatic embryonal carcinoma showed $10-25 \%$ of EGFR staining and $25-50 \%$ of EGFR staining, respectively.

\section{FISH Study for EGFR Gene Amplification}

EGFR gene amplification was performed on the metastatic embryonal components of all 21 cases. EGFR gene amplification was observed in one case (5\%). Polysomy (more than two copies of CEP7 and EGFR/CEP7 ratio $<2$ ) of chromosome 7 was observed in 15 of 21 cases $(71 \%)$ (Figure 1); the remaining 5 cases $(24 \%)$ exhibited a normal disomic FISH pattern.

Similarly, EGFR gene amplification was also evaluated on two primary testicular tumors available (one teratoma and one embryonal carcinoma). Both primary tumors showed EGFR polysomy, which is identical to retroperitoneal metastatic embryonal carcinoma component.

\section{Correlation between EGFR Protein Expression and Gene Amplification}

EGFR gene amplification status and protein expression level were compared in the metastatic embryonal components of all 21 cases. (Table 1) Positive EGFR protein expression was present in 9 of 21 cases (1 case had EGFR amplification, 7 cases had polysomy and 1 cases had disomy). EGFR protein expression was absent or very limited in 12 of 21 cases ( 8 patients had polysomy and 4 case had disomy). By contrast, EGFR gene amplification was observed in 1 of these 21 cases, with positive EGFR protein expression. Of 21 cases, 15 had polysomy (7 patients had positive EGFR protein 
10
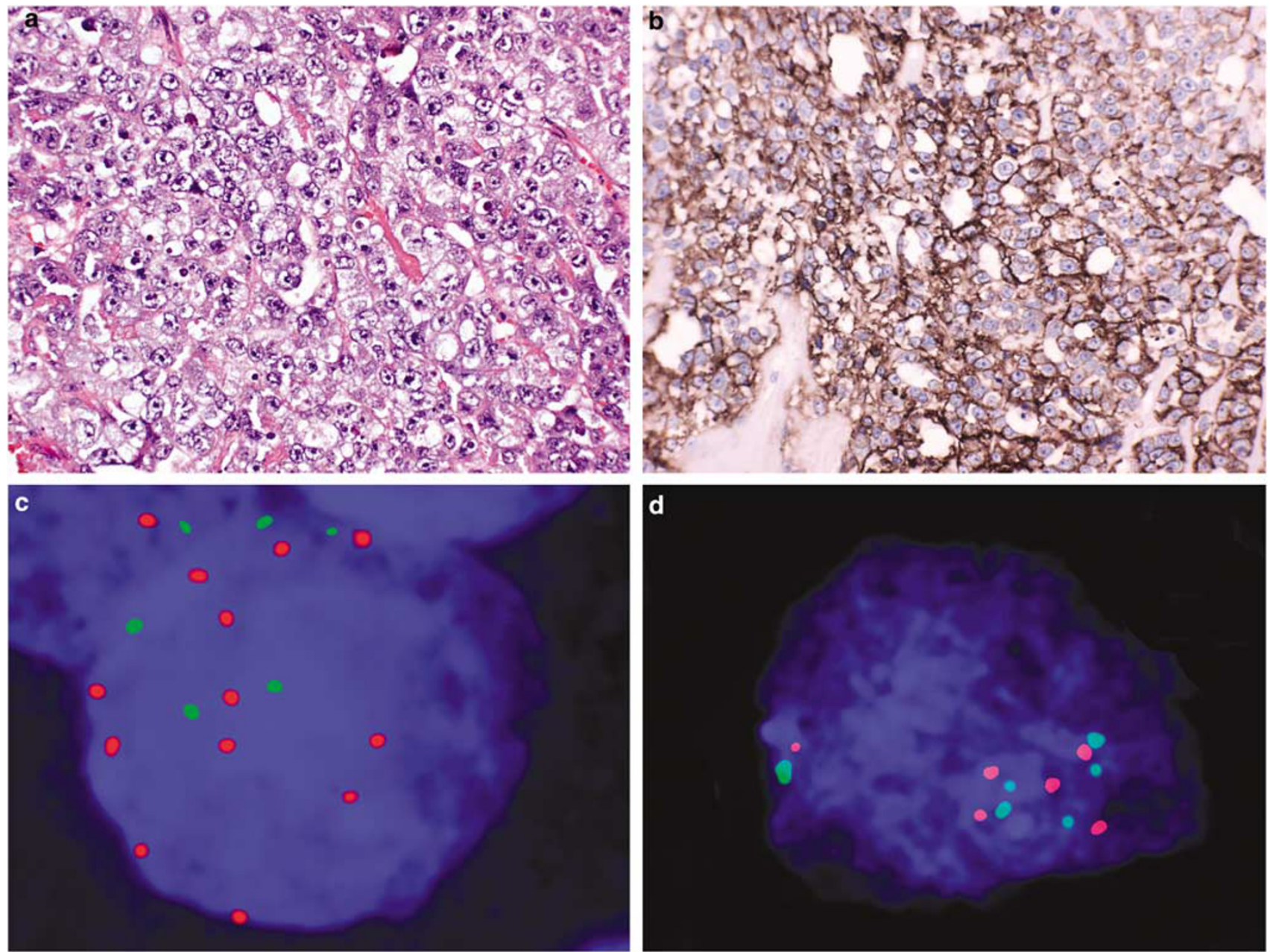

Figure 1 Epidermal growth factor receptor (EGFR) alterations in chemorefractory metastatic embryonal carcinoma. Metastatic embryonal carcinoma is composed of large epithelioid anaplastic cells in sheets (a). Immunohistochemistry showed EGFR expression in a mixed membranous and cytoplasmic distribution (b). EGFR gene alteration was detected by interphase fluorescence in situ hybridization (FISH) using LSI EGFR/CEP 7 Dual Color Probe containing centromere chromosome 7 (Spectrum Green) and EGFR (EGFR, Spectrum Orange). One case showed EGFR amplification as revealed by Red/Green (EGFR/CEP7) signal ratio >2 (c). Of 21, 15 cases showed polysomy pattern as indicated by > two green signals from CEP7 and Red/Green signal ratio below 2 (d).

Table 1 EGFR expression in metastatic chemorefractory embryonal carcinoma

\begin{tabular}{|c|c|c|c|}
\hline \multicolumn{2}{|c|}{ EGFR immunostaining } & \multicolumn{2}{|l|}{ EGFR FISH } \\
\hline$\%$ & No. of cases & Polysomy/amplification & Disomy \\
\hline 0 & 11 & 7 & 4 \\
\hline $1-10$ & 1 & 1 & 0 \\
\hline $10-25$ & 1 & 1 & 0 \\
\hline $25-50$ & 4 & 4 & 0 \\
\hline $50-75$ & 1 & 0 & 1 \\
\hline $75-100$ & 3 & $2 / 1$ & 0 \\
\hline Total & 21 & 16 & 5 \\
\hline
\end{tabular}

Abbreviations: EGFR, epidermal growth factor receptor; FISH, fluorescence in situ hybridization.

expression, 8 cases had no protein expression). The remaining five cases had disomy (one case had EGFR protein expression and four cases had no
EGFR protein expression). The results indicated a significant correlation between elevated EGFR protein expression level and EGFR gene polysomy/ amplification $(P=0.02)$.

\section{Discussion}

Testicular neoplasms account for $<1 \%$ of all malignancies in male patients. About 95\% of testicular cancers consist of germ cell tumors, with an estimated 7920 new cases and 380 deaths expected annually in the United States. On the basis of histological, biological, and clinical features, germ cell tumors are categorized into two groups: seminoma and nonseminomatous germ cell tumors. Nonseminomatous germ cell tumors are histologically heterogeneous and include one or more of the following components: teratoma (mature and/or immature), embryonal carcinoma, yolk sac 
tumor, and choriocarcinoma. Seminoma can coexist with other components in nonseminomatous germ cell tumors.

Germ cell tumors are typically managed initially by radical orchiectomy to establish the histological type of tumor and local tumor stage. Depending on clinical and pathological findings, subsequent management may include clinical observation, radiation therapy, RPLND, or cisplatin-based chemotherapy, and these treatments are curative in the great majority of patients. However, $30 \%$ of patients with disseminated disease at the time of initial diagnosis fail to achieve complete remission or subsequently relapse and require further salvage treatments, which may include high-dose chemotherapy with stem cell rescue. Approximately half of patients who suffer relapse eventually die of the disease. ${ }^{13}$

Chemotherapy resistance is an important biological and clinical issue in the management of germ cell tumors. The genetic pathways that determine chemotherapy sensitivity in germ cell tumors remain largely unknown. Patients who are chemotherapy resistant may have different genetic alterations. Chemoresistance has been linked to P53 gene mutations by some investigators ${ }^{14}$ but not others. ${ }^{15}$ By comparative genomic hybridization analysis, Rao et $a l^{16}$ identified possible amplified regions involving multiple chromosomes (1q, 2p, 7q, 9q, 9q, 15q, and 20q) in cisplatin-resistant germ cell tumors. Identification of amplified/overexpressed genes may elucidate genetic pathways of chemotherapy resistance in germ cell tumors and may help in the development of novel treatments targeted at the molecular level.

The EGFR superfamily (EGFR/erB-1, HER2/erB-2, HER3/erb-3, and HER4/erb-4) consists of different transmembrane growth factor tyrosine kinases that share similarities in structure and function and are important in cell survival and proliferation. EGFR (EGFR, HER-1, c-erbB-1) is expressed in many human epithelial malignancies. Overexpression of EGFR correlates with a poor prognosis and decreased survival in EGFR in some of these malignancies. ${ }^{17}$ The EGFR gene is located on chromosome 7 p12-p22 and encodes a $170 \mathrm{kDa}$ glycoprotein. Structurally, EGFR is composed of an extracellular EGF ligand-binding domain, a transmembrane lipophilic segment, and an intracellular tyrosine kinase domain. Two ligands can activate EGFR: epidermal growth factor and transforming growth factor. After the ligand binds EGFR, the receptors homodimerize or heterodimerize. Subsequently, tyrosine kinase of the receptor's intracytoplasmic domain is activated and signal pathways are triggered that may induce cellular growth, differentiation and proliferation, invasion, and increased metastatic potential of the tumor cells.

Currently, data concerning EGFR expression at the protein level or molecular level in metastatic embryonal carcinoma is very limited and available data are inconclusive. In a series of 88 testicular germ cell tumors, Hechelhammer et al ${ }^{18}$ observed EGFR reactivity only in the stromal cells of embryonal carcinoma (29\%), in the epithelial components of teratoma (71\%), and in syncytiotrophoblastic cells of other components. Moroni et al ${ }^{19}$ investigated 24 testicular tumors and detected EGFR staining in the $\beta$-HCG-positive components present in 16 of 18 nonseminomatous germ cell tumors, but observed no staining in the $\beta$-HCG-negative components of these tumors. In addition, they detected expression of EGFR activation (phosphorylated EGFR) in 27\% of the EGFR-positive tumors; their overall findings led them to suggest that active EGFR-targeted pharmaceutical agents may be clinically relevant in the management of mixed germ cell tumors with $\beta$-HCG expression. ${ }^{19}$ Kollmannsberger et $a l^{20}$ also demonstrated the presence of EGFR expression restricted to trophoblastic cells in 4 of 22 cisplatin-resistant germ cell tumors and in 3 of 12 chemosensitive germ cell tumors. In this study, only 4 of 22 chemoresistant tumors and 6 of 12 chemotherapy-responsive tumors included elements of embryonal carcinoma. In current study, we found $E G F R$ gene amplification in only 1 of 21 cases. However, we did demonstrate frequent polysomy, possibly representative of 'low level' gene amplification, in 15 of 21 cases (71\%). We also observed significant correlation between EGFR protein overexpression by immunohistochemical studies and gene amplification/polysomy by FISH.

The mechanisms underlying EGFR protein overexpression are not well understood and appear to differ markedly among various tumor types. Proposed mechanisms included gene amplification, activating mutations, increasing EGFR transcription or translation causing increased mRNA and protein production, decreased protein destruction, overexpression of receptor ligands, and others. Our results suggested that EGFR gene amplification/ polysomy appears to induce protein overexpression; however, EGFR protein expression may be influenced by other regulatory pathways, and further studies are needed to clarify this process. Significantly, our demonstration of frequent expression of EGFR in a subset of cases of metastatic chemorefractory embryonal carcinomas suggested that targeted therapy against EGFR (such as cetuximab or gefitinib) may be beneficial in such cases; this hypothesis is worthy of further investigation.

In summary, we found in our series of 21 patients with chemorefractory metastatic embryonal carcinoma that EGFR was expressed in 9 cases $(43 \%)$. EGFR gene polysomy/amplification were identified in 16 of 21 cases (76\%). To our knowledge, our study is the first to demonstrate EGFR gene amplification/ polysomy at the molecular level and the first to show a significant correlation between EGFR overexpression and gene amplification/polysomy in metastatic chemorefractory embryonal carcinoma. Our observation of EGFR expression in $43 \%$ of cases of chemorefractory metastatic embryonal carcinoma 
suggests that anti-EGFR therapy might be useful in the management of this subset of patients with such aggressive cancers. Further studies of larger sample focusing on specific histological subtypes as well as primary testicular tumors, including chemosensitive and chemorefractory tumors, are needed to define more clearly the prevalence and significance of EGFR expression in chemorefractory germ cell tumors.

\section{References}

1 Jemal A, Siegel R, Ward E, et al. Cancer statistics, 2008. CA Cancer J Clin 2008;58:71-96.

2 Lewis LG. Radioresistant testis tumors: results in 133 cases; five-year follow-up. J Urol 1953;69:841-844.

3 Einhorn LH. Treatment of testicular cancer: a new and improved model. J Clin Oncol 1990;8:1777-1781.

4 Grandis JR, Sok JC. Signaling through the epidermal growth factor receptor during the development of malignancy. Pharmacol Ther 2004;102:37-46.

5 Baselga J, Arteaga CL. Critical update and emerging trends in epidermal growth factor receptor targeting in cancer. J Clin Oncol 2005;23:2445-2459.

6 Stahtea XN, Roussidis AE, Kanakis I, et al. Imatinib inhibits colorectal cancer cell growth and suppresses stromal-induced growth stimulation, MT1-MMP expression and pro-MMP2 activation. Int J Cancer 2007;121:2808-2814.

7 Marshall J. Clinical implications of the mechanism of epidermal growth factor receptor inhibitors. Cancer 2006;107:1207-1218.

8 Dziadziuszko R, Hirsch FR, Varella-Garcia M, et al. Selecting lung cancer patients for treatment with epidermal growth factor receptor tyrosine kinase inhibitors by immunohistochemistry and fluorescence in situ hybridization-why, when, and how? Clin Cancer Res 2006;12:4409s-4415s.

9 Tsao MS, Sakurada A, Cutz JC, et al. Erlotinib in lung cancer-molecular and clinical predictors of outcome. N Engl J Med 2005;353:133-144.
10 Wang X, Zhang S, MacLennan GT, et al. Epidermal growth factor receptor protein expression and gene amplification in small cell carcinoma of the urinary bladder. Clin Cancer Res 2007;13:953-957.

11 Wang X, Jones TD, Zhang S, et al. Amplifications of EGFR gene and protein expression of EGFR, Her-2/neu, c-kit, and androgen receptor in phyllodes tumor of the prostate. Mod Pathol 2007;20:175-182.

12 Alvarez G, Perry A, Tan BR, et al. Expression of epidermal growth factor receptor in squamous cell carcinomas of the anal canal is independent of gene amplification. Mod Pathol 2006;19:942-949.

13 Sammler C, Beyer J, Bokemeyer C, et al. Risk factors in germ cell tumour patients with relapse or progressive disease after first-line chemotherapy: evaluation of a prognostic score for survival after high-dose chemotherapy. Eur J Cancer 2008;44:237-243.

14 Houldsworth J, Xiao H, Murty VV, et al. Human male germ cell tumor resistance to cisplatin is linked to TP53 gene mutation. Oncogene 1998;16:2345-2349.

15 Kersemaekers AM, Mayer F, Molier M, et al. Role of P53 and MDM2 in treatment response of human germ cell tumors. J Clin Oncol 2002;20:1551-1561.

16 Rao PH, Houldsworth J, Palanisamy N, et al. Chromosomal amplification is associated with cisplatin resistance of human male germ cell tumors. Cancer Res 1998;58:4260-4263.

17 Papadopoulou E, Tripsianis G, Anagnostopoulos K, et al. The influence of serum HER-2 levels and HER-2 codon 655 polymorphism on breast cancer outcome. Neoplasma 2008;55:113-121.

18 Hechelhammer L, Storkel S, Odermatt B, et al. Epidermal growth factor receptor is a marker for syncytiotrophoblastic cells in testicular germ cell tumors. Virchows Arch 2003;44:28-31.

19 Moroni M, Schiavo R, Carminati O, et al. Epidermal growth factor receptor expression and activation in nonseminomatous germ cell tumors. Clin Cancer Res 2001;7:2770-2775.

20 Kollmannsberger C, Nichols C, Bokemeyer C. Recent advances in management of patients with platinumrefractory testicular germ cell tumors. Cancer 2006; 106:1217-1226. 